\title{
Transient Posterior Encephalopathy Induced by Chemotherapy in Children
}

Rocío Sánchez-Carpintero, MD, PhD*, Juan Narbona, MD, PhD*, Reyes López de Mesa, MD*, Javier Arbizu, MD $\ddagger$, and Luis Sierrasesúmaga, MD, $\mathrm{PhD} \dagger$

From the *Pediatric Neurology Unit and †Pediatric Oncology Unit; Department of Pediatrics; and the $\ddagger$ Department of Nuclear Medicine; University Clinic of Navarra and School of Medicine; University of Navarra; Pamplona, Spain.

Communications should be addressed to:

Dr. Sánchez-Carpintero; Pediatric Neurology Unit; Department of Pediatrics; University Clinic of Navarra; P.O. Box 4209; Pamplona 31080, Spain.

\begin{abstract}
The cases of three children, 16, 12, and 12 years of age, who suffered sudden confusional state and cortical blindness lasting 12 to 30 minutes while under treatment with high-dose methotrexate, cyclophosphamide, and dactinomycin for a lower limb osteosarcoma are reported. Transient neuropsychologic deficits arose after the acute phase of treatment: left hemispatial neglect and constructive apraxia (Patient 1); constructive apraxia (Patient 2); and constructive apraxia and alexia without aphasia (Patient 3). The three patients recovered completely from all their deficits within the time frame of 3 hours to 2 weeks. Arterial hypertension and hypomagnesemia were found during the acute phase in all patients. In Patients 2 and 3, magnetic resonance imaging revealed increased parieto-occipital $\mathrm{T}_{2}$ signal involving gray and white matter. In Patients 1 and 2, HmPAO-SPECT revealed parieto-occipital hypoperfusion that resolved a few days later. The alterations detected by neuroimaging were concurrent with the appearance and disappearance of the clinical symptoms. Such transient acute episodes have been named occipital-parietal encephalopathy. On the basis of our clinical, laboratory, and neuroimaging findings, an explanation for the origin of this syndrome, a migrainelike mechanism, triggered by chemotherapy-induced hypomagnesemia, is proposed.
\end{abstract}




\section{INTRODUCTION}

Complicated migrainelike episodes during immunosuppressive therapy or antitumoral chemotherapy with or without cranial irradiation have been described in adult $[1,2]$ and pediatric patients $[3,4]$. In general the acute clinical features include confusional state, loss of vision, headache, and, in many cases, epileptic seizures. Although the physiopathology of this syndrome remains unclear, a reversible brain ischemic phenomenon has been proposed that could be caused by arterial hypertension, low $\mathrm{Mg}^{2+} / \mathrm{Ca}^{2+}$ ratio, and brain microvascular anatomophysiologic changes induced by chemotherapy and/or radiotherapy. On the basis of neuroimaging features, the names reversible posterior leukoencephalopathy [1] and occipital-parietal encephalopathy [4] have been proposed for this syndrome.

Three pediatric patients with osteosarcomas in their legs who suffered episodes of this transient encephalopathy during chemotherapy without previous cranial irradiation are reported. On the basis of their clinical features, biochemical tests, and morphologic/functional neuroimaging, the physiopathology and possible therapeutic management of this syndrome are discussed.

\section{CASE REPORTS}

\section{Patient 1}

This patient was a 16-year-old male with a fibular osteogenic sarcoma who initially received high-dose methotrexate (HDMTX) chemotherapy (T10 Rosen-modified protocol) that was discontinued 1 month before completion because of prolonged toxic serum levels. The patient was then put on cyclophosphamide and dactinomycin, but 1 day later he developed an acute confusional state with motor restlessness, dystonic limb spasms, and headache, all of which lasted 30 minutes. Then cortical blindness developed for several minutes, followed by residual left homonymous hemianopsia and hemiparesia; he demonstrated constructive apraxia and partial left hemispatial neglect during the copy of the Rey-Osterrieth complex figure. Thirty-six hours later the motor and visual hemisyndrome had disappeared entirely, the patient did not present any symptoms of visual agnosia or negligence, and his copy of the Rey figure was normal.

His initial blood pressure was 150/90 $\mathrm{mm} \mathrm{Hg}$, and his serum total magnesium level was $1.3 \mathrm{mg} / \mathrm{dL}$ (normal range 1.9-2.5 mg/dL). A computed tomography scan did not reveal metastasis or other lesions. During the acute stage the electroencephalogram (EEG) was characterized by hypersynchronous slow waves. A brain singlephoton emission computed tomography (SPECT) using ${ }^{99 \mathrm{~m}} \mathrm{Tc}-\mathrm{HmPAO}$ (Ceretec, Nycomed-Amershan) was obtained and revealed hypoperfusion on the right parieto-temporo-occipital region (Fig 1A). Thirty-six hours after initial presentation, when clinical symptoms had completely resolved, EEG and a new brain ${ }^{99 \mathrm{~m}}$ Tc-HmPAO SPECT (Fig 1B) revealed no abnormality. Treatment was started during the acute phase. Restoration of normal magnesium serum levels $(1.9-2.4 \mathrm{mg} / \mathrm{dL})$ was achieved in 24 hours after IV perfusion of maintenance doses of $3 \mathrm{mg} / \mathrm{kg} /$ day of magnesium sulphate. Oral flunarizine (10 mg, single dose) and dexamethasone (1 mg/kg per day for 1 week) treatment was initiated. 


\section{Patient 2}

The second patient was a 12-year-old male with a right femur osteosarcoma who first had received IV HDMTX infusion, which was later replaced by a cycle of cyclophosphamide, bleomycin, dactinomycin, and vincristine. One week later he suddenly presented slurred speech, disorientation, and dystonic posture of his right arm with head deviation to the right; this episode lasted for 2-3 minutes. A homonymous left hemianopsia was later confirmed in the emergency department, where the patient then presented a generalized tonic-clonic seizure that ended 25 minutes later with rectal diazepam and a loading dose of IV phenytoin. Hemianopsia resolved in 24 hours. Then the patient demonstrated a constructive apraxia with the Rey-Osterrieth complex figure test, from which he recovered 2 days later. Blood pressure at admission was 150/100 $\mathrm{mm} \mathrm{Hg}$ and serum magnesium level was $1.9 \mathrm{mg} / \mathrm{dL}$, which is at the lower end of the normal range. EEG hypersynchronous slow waves were present in the right posterior area. Magnetic resonance imaging (MRI) of the brain 2 hours after admission revealed a high $\mathrm{T}_{1}$ and $\mathrm{T}_{2}$ corticosubcortical signal on the right temporo-occipital junction. The ${ }^{99 \mathrm{~m}}$ Tc-HmPAO SPECT 6 hours after the start of symptoms exhibited global hypoperfusion of the right cerebral hemisphere, more marked in premotor and temporooccipital areas.

Three days later, MRI scan and brain SPECT were completely normal. Maintenance oral doses of magnesium lactate (12 $\mathrm{mg} / \mathrm{kg}$ per day of magnesium element) restored the normal serum levels. Oral dexamethasone ( $1 \mathrm{mg} / \mathrm{kg}$ per day) was given for 1 week and a single dose of oral flunarizine (10 mg) was given during the acute phase.

\section{Patient 3}

The third patient was a 12-year-old female suffering from a right tibial sarcoma who had received her first IV HDMTX cycle followed by cyclophosphamide, dactinomycin, bleomycin, and vincristine IV infusion. Six days later, she developed sudden cortical blindness and headache with occasional vomiting for 2 hours. These symptoms were followed by a generalized tonic-clonic seizure. After recovery, visual agnosia developed. Blood pressure was 160/120 mm Hg, serum magnesium level was 1.5 $\mathrm{mg} / \mathrm{dL}$, and serum creatinine was $1.5 \mathrm{mg} / \mathrm{dL}$ (normal range is $0.1-1.2 \mathrm{mg} / \mathrm{dL}$ ).

Subsequently, every 2 weeks the patient underwent neuropsychologic tests that revealed constructive apraxia and alexia without aphasia, both of which resolved in 3 months. A generalized low-voltage slow rhythm was seen on the EEG. A cerebral MRI scan indicated bilateral white and gray matter changes on parieto-temporo-occipital areas, more intense on the left side (Fig 2), which disappeared within 3 months. Functional neuroimaging was not performed. Treatment with oral magnesium (12 mg/kg per day) led to the restoration of normal serum levels within the first 24 hours after admission. Dexamethasone (1 mg/kg per day) was given for 1 week. 


\section{DISCUSSION}

Three patients are reported who developed an acute onset of confusional state and cortical blindness lasting up to 30 minutes while they were treated with HDMTX, cyclophosphamide, and dactinomycin. A similar transient acute encephalopathy has been reported as a side effect in patients receiving chemotherapy or immunosuppressive treatment $[1,2,4]$. Despite some evidence of gray matter changes demonstrated by MRI scan, the syndrome was termed reversible posterior leucoencephalopathy by Hinchey et al. [1]. MR spectroscopy and positron emission tomography (PET) findings reported by Pavlakis et al. [4] in children demonstrated neuronal damage, so the term occipitalparietal encephalopathy, which includes white matter changes and gray matter damage, was proposed. We have chosen to refer to this syndrome as posterior encephalopathy.

The mechanisms underlying this condition remain unclear. A hypertensive encephalopathy-like mechanism, in addition to the toxic effect of the drugs on the endothelium of the blood-brain barrier, has been proposed as a possible explanation by Hinchey et al. [1]. They reported a long series in adults and postulated the physiopathologic mechanism, but blood pressure was normal in one third of the patients included in the study. The arterial pressure values in Patients 1 and 2 of our study give little support to this theory because they are far from those values needed to provoke a true hypertensive encephalopathy [5]. In addition, brain SPECT has demonstrated localized hyperperfusion in subjects suffering from hypertensive encephalopathy [6] in contrast to the hypoperfusion found in our patients.

Considering the clinical, SPECT, and laboratory findings in our patients, a migrainelike mechanism may be proposed. Clinical symptomatology includes transient development of confusion, agitation, seizures, and transient cortical blindness followed by reversible neuropsychologic deficits. Patients 1 and 2 recovered spontaneously within a few days from all clinical symptoms and from alterations detected by morphologic and functional neuroimaging. Patient 3 also recovered rapidly, but a residual reading disorder took 2 months to resolve completely. This clinical symptomatology is similar to that seen in patients with complicated migraine [7]. Thus we think that this type of transient acute encephalopathy may be due to a similar brain disorder.

The posterior brain region hypoperfusion demonstrated by SPECT in Patients 1 and 2 also supports this migrainelike theory. Others also have been able to describe hypoperfusion with SPECT during the aura phase of the migraine, mainly in parietooccipital regions [8]. On the contrary, hyperperfusion has been found with SPECT in hypertensive encephalopathy [6].

The present theory about the pathophysiology of the migraine aura is based on the cortical spreading depression (CSD) phenomenon, which is thought to be responsible for the negative neurologic symptoms [9]. The CSD is a short-lasting depolarization wave that moves across the cortex provoking a prolonged nerve cell depression. The hypoperfusion that has been demonstrated by SPECT is thought to be a secondary event because of the involvement of arterioles in the homeostatic brain changes occurring during the spreading depression [9].

The initiating and underlying causes of the CSD are not yet fully understood. It is known that glutamate can trigger CSD by activating N-methyl-D-aspartate receptors and 
that low brain magnesium strongly facilitates the elicitation of CSD in vitro. For this reason, the laboratory finding of low magnesium serum levels in the patients reported here may also support the migrainelike mechanism for this posterior encephalopathy. Several studies have reported low levels of plasma total and ionized magnesium during and between migraine attacks, so an imbalance in the $\mathrm{Ca}^{2+} / \mathrm{Mg}^{2+}$ ratio has been postulated as a triggering mechanism of transient vasomotor instability $[10,11]$. Therapeutic trials with magnesium sulfate have been successful in diminishing the frequency of cephalalgic episodes and in aborting attacks [12,13]. In all three patients, total magnesium plasma levels were below normal limits. Hypomagnesemia occurs frequently during cancer chemotherapy y; MTX in association with cyclophosphamide or iphosphamide causes a renal tubulopathy that leads to $\mathrm{Mg}^{2+}$ loss in $70 \%$ of patients [14].

Neither previous episodes of severe intermittent headache that could be diagnosed as migraine nor family history of migraine were reported by our patients. Family predisposition is not, therefore, likely to be a triggering mechanism of the episodes in these three patients. Therefore low magnesium serum levels could be a trigger instead. As suggested by Hinchey et al. [1] a vascular instability resulting from the toxicity of the chemotherapy agents on the endothelium of the blood-brain barrier can also be contributing. A multifactorial origin of the disorder is more likely because not every patient with low magnesium serum levels while receiving chemotherapy develops this encephalopathy.

Other theories have been proposed to explain the physiopathology of this acute posterior encephalopathy. Micrometastasis or necrotic tissue embolisms from lungs are not probable because of the transient symptoms and the absence of lung metastasis in all of the cases reported here and by others [15].

In the literature, this acute posterior encephalopathy most frequently is associated with HDMTX treatments. However, it is not clear which drug was responsible in the cases reported here. HDMTX had been administered 1 week before the episodes in Patients 2 and 3, but it had not been given for 1 month, because of toxicity, in Patient 1 . The three patients had also received cyclophosphamide and dactynomycin within the few days preceding the neurologic complication.

In conclusion, clinical, neuroimaging, and laboratory observations support the hypothesis that a migrainelike mechanism underlies this acute encephalopathic syndrome sometimes observed during aggressive chemotherapy. If sudden-onset neurologic deficits arise and brain hemorrhage, central nervous system infection, and hydroelectrolytic disturbances have been excluded, this posterior encephalopathy can be diagnosed. Arterial hypertension may be a precipitant in some cases together with endothelium brain barrier damage; however, $\mathrm{Ca}^{2+} / \mathrm{Mg}^{2+}$ imbalance is probably the most important trigger of vasomotor instability and of the CSD phenomenon. Oral magnesium prophylaxis is a common practice in patients receiving chemotherapy, but anorexia and/or vomiting sometimes make it impossible to achieve normal serum levels. Calcium channel antagonists and magnesium sulphate, perhaps in addition to corticosteroids for brain edema and other antihypertensive agents if needed, might shorten the symptomatic period. 


\section{REFERENCES}

[1] Hinchey J, Chaves C, Appignani B, et al. A reversible posterior leukoencephalopathy syndrome. N Engl J Med 1996;334:494-500.

[2] Grivé E, Rovira-Caellas A, Acebedo G, Alvarez-Sabin J. Leucoencefalopatía reversible relacionada con el tratamiento con ciclosporina. Rev Neurol 1997;25:460-4.

[3] Shuper A, Packer RJ, Vezina LG, Nicholson HS, Lafond D. “Complicated migraine-like episodes" in children following cranial irradiation and chemotherapy. Neurology 1995;45:1837-40.

[4] Pavlakis SG, Frank Y, Kalina P, Chondra M, Lu D. Occipital-parietal encephalopathy: A new name for an old syndrome. Pediatr Neurol 1997;16:1458.

[5] Hulse JA, Taylor DST. Blindness and paraplegia in severe childhood hypertension. Lancet 1979;2:553-5.

[6] Schwarz RB, Jones KM, Kalina P, et al. Hypertensive encephalopathy: Findings on CT, MR imaging and SPECT imaging in 14 cases. AJR 1992;159:379-83.

[7] Chumbe G, Narbona J, Villa-Eizaga I. Migraña confusional en la infancia. Acta Pediatr Esp 1990;48:42-4.

[8] De Benedittis G, Ferrari Da Passano C, Granata G, Lorenzetti A. CBF changes during headache-free periods and spontaneous/induced attacks in migraine with and without aura: A TCD and SPECT comparison study. J Neurosurg Sci 1999;43:141-6.

[9] Lauritzen M. Pathophysiology of the migraine aura. The spreading depression theory. Brain 1994;117:199-210.

[10] Soriani S, Arnaldi C, De Carlo L, et al. Serum and red blood cells magnesium levels in juvenile migraine patients. Headache 1995;35: 14-6.

[11] Welsh RMA, Schultz L, Nelson J. Reduced Mg++ in hemiplegic migraine not observed in migraine with aura or without aura using P31 magnetic resonance spectroscopic imaging. Cephalalgia 1999;19: 310 (abstr).

[12] Peikert A, Wilimzig C, Kohne-Volland R. Prophylaxis of migraine with oral magnesium: Results from a prospective, multi-center, placebo-controlled and double-blind randomised study. Cephalalgia 1996;16:257-63.

[13] Wilson MC, Williams M. Intravenous magnesium as a treatment of migraine in pregnancy. Cephalalgia 1999;19:379 (abstr).

[14] Michelagnoli MP, Lewis IJ, Gattamaneni HR, Bailey CC, Lashford LS. Ifosfamide/etoposide alternating with high-dose methotrexate: evaluation of a chemotherapy regimen for poor-risk osteosarcoma. Br J Cancer 1999;79:1174-8.

[15] Walker RW, Allen JC, Rosen G, Caparros B. Transient cerebral dysfunction secondary to high-dose methotrexate. J Clin Oncol 1986;4: 1845-50. 


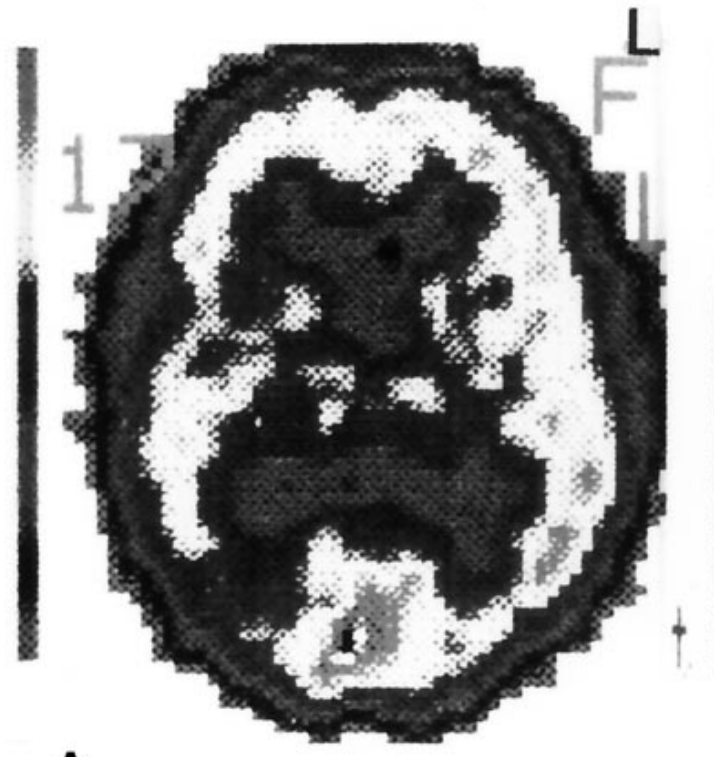

A

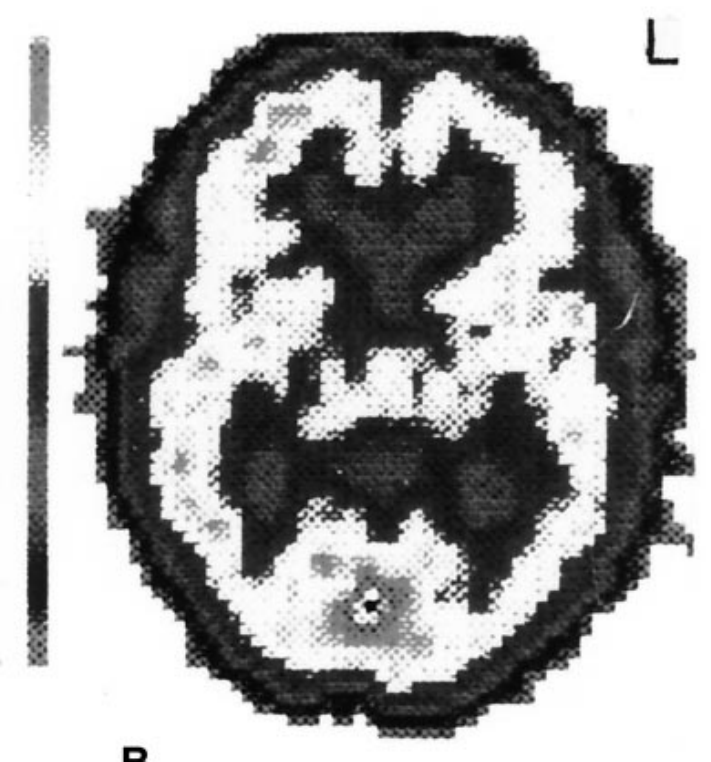

B

Figure 1. (A) ${ }^{99 \mathrm{~m}} \mathrm{Tc}-\mathrm{HmPAO}$ SPECT of Patient 1 during the acute stage demonstrating hypoperfusion in right hemisphere, predominantly in white matter, but also affecting gray matter in the parieto-occipital area. (B) ${ }^{99 \mathrm{~m}} \mathrm{Tc}-\mathrm{HmPAO}$ SPECT 36 hours after the acute episode revealed normal perfusion.

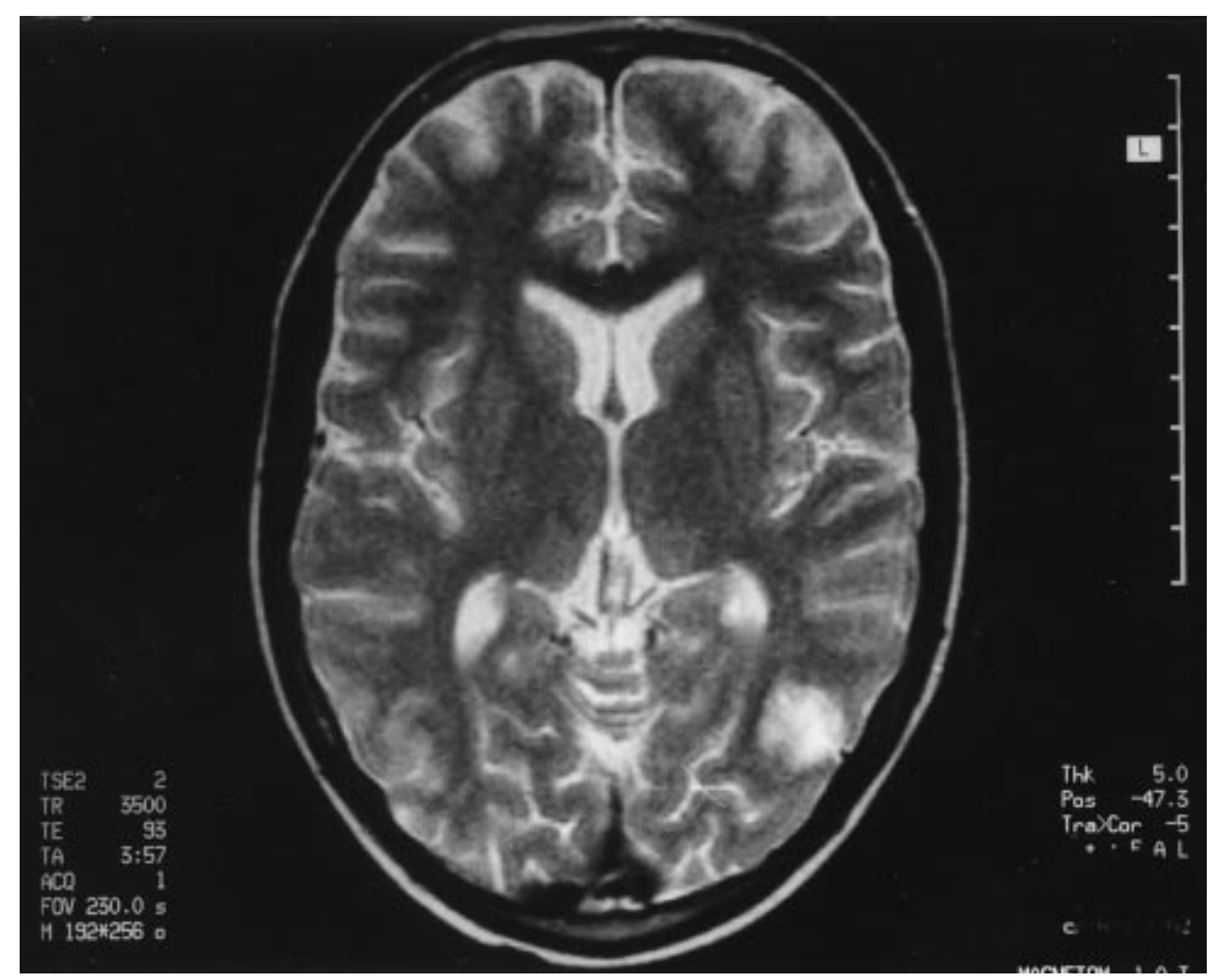

Figure 2. MRI brain scan findings of Patient 3. Axial $\mathrm{T}_{2}$-weighted image demonstrates hyperintense signal involving parieto-temporo-occipital areas $\left(\mathrm{T}_{2}\right.$ 3500/93/1 (TR/TE/ADQ). FOV: 250. Matrix: 192 x 256. Thickness: 5 mm. 1 T). 